TTP07-33, SFB/CPP-07-79

\title{
Naturalness and the Neutrino Matrix
}

\author{
J. Sayre ${ }^{1}$ and S. Wiesenfeldt ${ }^{1,2}$ \\ ${ }^{1}$ Department of Physics, University of Illinois at Urbana-Champaign, \\ 1110 West Green Street, Urbana, IL 61801, USA \\ ${ }^{2}$ Institut für Theoretische Teilchenphysik, Universität Karlsruhe, \\ 76128 Karlsruhe, Germany
}

\begin{abstract}
The observed pattern of neutrino mass splittings and mixing angles indicates that their family structure is significantly different from that of the charged fermions. We investigate the implications of these data for the fermion mass matrices in grand unified theories with a type-I seesaw mechanism. We show that, with simple assumptions, naturalness leads to a strongly hierarchical Majorana mass matrix for heavy right-handed neutrinos and a partially cascade form for the Dirac neutrino matrix. We consider various model building scenarios which could alter this conclusion, and discuss their consequences for the construction of a natural model. We find that including partially lopsided matrices can aid us in generating a satisfying model.
\end{abstract}

\section{Introduction}

The measurement of neutrino mass splittings and mixing angles [1, 2] has provided a new window into physics beyond the Standard Model. The fact that the hierarchy between at least one pair of the neutrinos is weak and that two leptonic mixing angles are large, in contrast to the strongly hierarchical masses of quarks and charged leptons and small CKM mixing, was initially surprising. It leads us to surmise that neutrino masses arise through a somewhat different mechanism than the quark and charged lepton masses. Thus, the relation between the charged fermion and neutrino observables is not necessarily obvious. In fact, we have such a mechanism in the form of the type-I seesaw [3], which can naturally yield neutrino masses in the range indicated by experiment. Moreover, the physical light neutrino mass matrix is a product of more fundamental matrices. This fact can potentially explain the differences between the mixing angles and mass hierarchies of the charged fermion and neutrino sectors.

The seesaw mechanism arises naturally within a grand-unified theory (GUT) such as $\mathrm{SO}(10)$ [4], where each generation of standard model fermions is unified into the 16-dimensional spinor 
representation, together with the right-handed neutrinos. The breaking of $B-L$ (where $B$ and $L$ denote baryon and lepton number, respectively), which is a subgroup of $\mathrm{SO}(10)$, automatically gives rise to Majorana masses for the singlet neutrinos, and thence to the seesaw mechanism. Indeed, the neutrino data have encouraged GUT model building [5, 6].

Although GUTs provide a natural framework for massive neutrinos and, combined with family symmetries or textures, have allowed for a number of successful models of quark masses and mixing, it has proven difficult to incorporate neutrinos in a completely satisfactory manner. In this paper, we reconsider neutrino masses and mixings under the guidance of naturalness. That is, rather than focusing on a particular theoretical structure and modifying it as necessary to obtain the best fit to the data, we will try to minimize the dependence on specific model assumptions and work up from the experimental data to see where it naturally leads us. In particular, we will show that the construction of a natural, unified picture of all standard model fermion masses and mixing angles imposes non-trivial constraints on the structure of both sectors.

In this framework, we are interested only in the orders of magnitude of various parameters and, in pursuing natural solutions, we seek to avoid unnatural cancellations, i.e., that terms of a given order must cancel to produce a term of lower order. It may be possible to arrange such cancellations in a technically natural way via a judicious choice of symmetries, but this is by no means trivial. Furthermore, an exact symmetry is a strong assumption to make, given the current uncertainty in the neutrino data. We will instead adopt naturalness as described above, seeking to constrain the approximate structure of our theory without ad hoc symmetries. Ideally, this structure can serve as a guide for developing well-motivated symmetries upon which an ultimately satisfying theory can be built.

Of course, one must make some assumptions based on previous successes to make progress and, in this capacity, we will focus on the $\mathrm{SO}(10)$ models with small representations [7, 8]. This scenario will serve as a concrete example; however, much of the analysis could be adapted to $\mathrm{SO}(10)$ models with large representations and/or type-II seesaw mechanisms, as well as to other unifying groups.

This paper is organized as follows: We start by introducing our theoretical framework in Section 2 and reviewing the experimental data in Section 3. In Section 4 we derive natural constraints on the neutrino mass matrices. Since the fermion mass matrices are related by the GUT symmetry, we study the implications of quark mixing in Section 5. In Section 6 we show how mass matrices consistent with our constraints can be generated via family symmetries, and we investigate how well they can fit the charged fermion masses. In SO(10) models with small representations, the neutrino Dirac mass matrix can receive additional contributions via couplings to a second up-type Higgs doublet, present in the $B-L$ breaking Higgs field. We consider this possibility in Section 7, supplemented by an Appendix. The remaining sections are devoted to two cases which generalize beyond our initial assumptions. These involve models wherein otherwise negligible leptonic rotations play an important role in neutrino mixing, either due to a lopsided structure in some mass matrices (Section 8), or to a particular form for the effective neutrino matrix (Section 9). We conclude in Section 10. 


\section{General Stucture of Theory}

The standard model fermions are found in three copies of the spinor representation $\mathbf{1 6}_{i}$. 1 We will make use of the small representations $\mathbf{1 0}_{H}, \mathbf{4 5} \mathbf{5}_{H}, \mathbf{1 6}_{H}, \mathbf{1 6}_{H}^{\prime}, \overline{\mathbf{1 6}}_{H}$, and potentially $\overline{\mathbf{1 6}}_{H}^{\prime}$ to break the GUT symmetry and to generate fermion masses. Several authors have used this framework to build interesting models [7, 8].

The SO(10) symmetry is broken to the Standard Model by GUT scale vacuum expectation values (vevs), one in the $\mathrm{SU}(5)$ singlet direction of $\mathbf{1 6}_{H}$ and $\overline{\mathbf{1 6}}_{H}$, denoted $v$, and $\left\langle\mathbf{4} \mathbf{5}_{H}\right\rangle$ along the $B-L$ direction. The electroweak symmetry is broken when weak doublets in $\mathbf{1 0}_{H}$ acquire vevs. It is also possible that the doublets in $\mathbf{1 6}_{H}^{\prime}$ and $\overline{\mathbf{1 6}}_{H}^{\prime}$ acquire weak scale vevs, in which case the light Higgs doublets are a mixture of weak doublets from the vector and spinor representations 9]. We will assume for now that $\overline{\mathbf{1 6}}_{H}^{\prime}$ does not acquire a weak vev.

Charged fermion masses are generated via several operators: the renormalizable operator $\mathbf{1 6}_{i} \mathbf{1 6}_{j} \mathbf{1 0}_{H}$, which contributes to all Dirac mass matrices for the standard model fermions; the higher-dimensional operator $\mathbf{1 6}_{i} \mathbf{1 6} \mathbf{6}_{j} \mathbf{1 0} \mathbf{4 5}_{H}$, which differentiates the quark mass matrices from the lepton matrices due to their differing charges under $B-L$; and $\mathbf{1 6}_{i} \mathbf{1 6}_{j} \mathbf{1 6}_{H} \mathbf{1 6}_{H}^{\prime}$, which contributes only to down quark and charged lepton mass matrices. The operator $\mathbf{1 6}_{i} \mathbf{1 6}_{j} \mathbf{1 0}_{H}$ is symmetric in generation space while $\mathbf{1 6}_{i} \mathbf{1 6}_{j} \mathbf{1 0}_{H} \mathbf{4 5}_{H}$ is antisymmetric $\left(\mathbf{1 6}_{i}\right.$ and $\mathbf{1 6}$ are contracted as a 120, for $\langle 45\rangle \propto B-L$ this is the only contraction that contributes to the mass matrices). The operator $1 \mathbf{6}_{i} \mathbf{1 6}_{j} \mathbf{1 6}_{H} \mathbf{1 6}_{H}^{\prime}$ may be symmetric or asymmetric, depending on how the fields are contracted.

With this set of operators, the Dirac neutrino matrix $M_{D}$ receives contributions from the operators $16_{i} \mathbf{1 6}_{j} \mathbf{1 0}_{H}$ and $1 \mathbf{6}_{i} \mathbf{1 6}_{j} \mathbf{1 0}_{H} \mathbf{4 5}_{H}$, and we expect it to be somewhat similar to the up quark matrix, i.e., to have a similarly strong hierarchy of mass eigenstates from the first to the third generation. For the up quarks this is approximately five orders of magnitude. Although the neutrino hierarchy can be somewhat weaker due to factors of 3 coming from the $B-L$ direction vev of the $4 \mathbf{5}_{H}$, one would still expect roughly a $10^{-4}$ ratio between the lightest and heaviest Dirac matrix eigenvalues.

We define the orientation of $M_{D}$ as $\nu^{i} M_{D}^{i j} N^{j}$, where $N$ is the Standard Model singlet. Then we can parameterize the Dirac matrix as

$$
M_{D} \equiv L_{D} D_{D} R_{D}^{\dagger}
$$

Here and throughout the paper the matrices $M$ are dimensionless and the largest eigenvalue is normalized to 1 . Since we are primarily concerned with interfamily relations this causes no problems, but one should bear in mind that there is an overall scale associated with all mass matrices. In the above case, the dimensionful Dirac mass operator is $u \nu M_{D} N$, where $u$ is the mass of the largest eigenvalue. Similarly, throughout the paper $L$ and $R$ will signify unitary

\footnotetext{
${ }^{1}$ The subscripts $i, j$ will be used to indicate generations while Higgs fields will be denoted with a subscript $H$.
} 
matrices defined by the diagonalization equations

$$
L^{\dagger} M M^{\dagger} L=R^{\dagger} M^{\dagger} M R=D^{2} \equiv \operatorname{diag}\left(\eta^{2}, \epsilon^{2}, 1\right),
$$

where $\eta, \epsilon, 1$ are the normalized eigenvalues of $M$.

In general, $L_{D}$ and $R_{D}$ are arbitrary unitary matrices and $D_{D}$ is a diagonal matrix of the eigenvalues of $M_{D}$; however, we expect the eigenvalues to be strongly hierarchical. This hierarchy will be naturally generated if we posit the forms

$$
D_{D} \equiv \operatorname{diag}(\eta, \epsilon, 1), \quad L_{D} \sim\left(\begin{array}{ccc}
1 & \mu^{\prime} \sqrt{\frac{\eta}{\epsilon}} & \nu^{\prime} \sqrt{\eta} \\
\mu^{\prime} \sqrt{\frac{\eta}{\epsilon}} & 1 & \rho^{\prime} \sqrt{\epsilon} \\
\nu^{\prime} \sqrt{\eta} & \rho^{\prime} \sqrt{\epsilon} & 1
\end{array}\right), \quad R_{D} \sim\left(\begin{array}{ccc}
1 & \mu \sqrt{\frac{\eta}{\epsilon}} & \nu \sqrt{\eta} \\
\mu \sqrt{\frac{\eta}{\epsilon}} & 1 & \rho \sqrt{\epsilon} \\
\nu \sqrt{\eta} & \rho \sqrt{\epsilon} & 1
\end{array}\right) .
$$

We expect $\eta \ll \epsilon \ll 1$. Based on the quark hierarchy we may estimate their approximate size as $\eta \sim 10^{-4}$ and $\epsilon \sim 10^{-2}$, but most of the analysis does not depend on this assumption.

$L_{D}$ and $R_{D}$ are unitary matrices and the parameterizations above should be read as giving the orders of magnitude only of the various entries. The parameters $\mu, \nu, \rho$ and their primed counterparts are generally expected to be less than or equal to order one. If they were significantly larger, various entries would need to cancel to preserve the smaller eigenvalues. Thus $\mu, \nu, \rho \sim 1$ is the minimal requirement for naturalness in the absence of an exact symmetry relating the Yukawa couplings. This is known as a geometrical hierarchy pattern [10]. It corresponds to the following form for $M_{D}$ :

$$
M_{D} \sim\left(\begin{array}{ccc}
\leq \eta & \sqrt{\eta \epsilon} & \sqrt{\eta} \\
\sqrt{\eta \epsilon} & \leq \epsilon & \sqrt{\epsilon} \\
\sqrt{\eta} & \sqrt{\epsilon} & 1
\end{array}\right)
$$

The central feature of such a matrix is that the off-diagonal entries play a dominant or codominant role in determining the two smaller eigenvalues. A geometric hierarchy can be easily obtained with a $U(1)$ symmetry via the Froggatt-Nielsen mechanism [11].

On the other hand, $\mu, \nu$, and $\rho$ may be arbitrarily smaller without endangering the eigenvalue hierarchy. In this case the diagonal entries in $M_{D}$ become dominant and must be correspondingly close to the eigenvalues. We will refer to this possibility as a sub-geometric hierarchy. With three generations it is, of course, possible to have a mixed case which is partially geometric and partially sub-geometric.

There is one exception to these naturalness considerations, which occurs if $M_{D}$ is highly asymmetric, i.e., if $\left(M_{D}\right)_{j i}$ and $\left(M_{D}\right)_{i j}$ are of different orders for some $i$ and $j$. However, if it arises only from $1 \mathbf{1 6}_{i} \mathbf{1 6} \mathbf{1 0}_{H}$ and $\mathbf{1 6}_{i} \mathbf{1 6}_{j} \mathbf{1 0}_{H} \mathbf{4 5} \mathbf{5}_{H}$, we would not expect this; these operators give symmetric and antisymmetric contributions, respectively, which would have to be arranged to cancel in a seemingly unnatural way. Thus we generally expect $L_{D}$ and $R_{D}$ to have similar values for their parameters, i.e., $\mu \sim \mu^{\prime}, \nu \sim \nu^{\prime}$ and $\rho \sim \rho^{\prime}$.

To implement the Type-I seesaw, we need a matrix for the heavy neutrinos: $N^{i} M_{R}^{i j} N^{j}$. Such a coupling may arise from $\frac{1}{m}\left(M_{R}\right)_{i j} \mathbf{1 6}_{i} \mathbf{1 6}_{j} \overline{\mathbf{1 6}}_{H} \overline{\mathbf{1 6}}_{H}$ when $\overline{\mathbf{1 6}}_{H}$ acquires its GUT scale vev $v$. This 
non-renormalizable operator is suppressed by some mass $m$, which is by default the Planck scale but which in practice may be somewhat less, depending on the origin of the effective operator. The seesaw formula then gives

$$
M_{\nu} \simeq-M_{D} M_{R}^{-1}\left(M_{D}\right)^{T}
$$

As discussed above, $M_{D}, M_{\nu}$ and $M_{R}$ are dimensionless. The massive parameter which sets the scale for the neutrinos is $u^{2} m / v^{2}$. For $u \sim 100 \mathrm{GeV}, v \sim 10^{16} \mathrm{GeV}$, and $m \sim m_{\mathrm{Pl}} \sim 10^{18} \mathrm{GeV}$, this comes out to be $0.1 \mathrm{eV}$, consistent with the range indicated by experiment.

We stress that the discussion above depends very little on the assumption of small representations or the vevs used to do symmetry breaking. One may for example use $\left\langle\mathbf{4} \mathbf{5}_{H}\right\rangle$ proportional to the hypercharge generator or use a $\mathbf{5} \mathbf{4}_{H}$ in place of the $\mathbf{4 5}_{H}$ to accomplish the breaking from $\mathrm{SU}(5)$ to the standard model [12]. Alternatively, we could have used the large representation approach with $\mathbf{1 0}_{H}, \mathbf{1 2 0}_{H}$, and $\mathbf{1 2 6} \mathbf{6}_{H}$, which many authors have used for model building [13]. In any case, we still expect a hierarchy in the quark and charged lepton mass matrices. Due to $\mathrm{SO}(10)$ relations, this hierarchy should manifest itself in the Dirac neutrino matrix as well and the same naturalness considerations apply.

\section{Experimental Constraints}

The detection of neutrino oscillation is successfully explained by massive neutrinos with nontrivial mixing. We know two mass squared splittings among the neutrinos and two mixing angles of the leptonic mixing matrix, with a limit on the third for the physical light neutrinos [2],

$$
\begin{array}{rlrl}
\tan ^{2} \theta_{12} & =0.45 \pm 0.05 ; & \Delta m_{\mathrm{sol}}^{2} & \simeq(8.0 \pm 0.3) \times 10^{-5} \mathrm{eV}^{2} \\
\sin ^{2} 2 \theta_{23} & =1.02 \pm 0.04 ; & \Delta m_{\mathrm{atm}}^{2} \simeq(2.5 \pm 0.2) \times 10^{-3} \mathrm{eV}^{2} \\
\sin ^{2} 2 \theta_{13} & =0 \pm 0.05 . &
\end{array}
$$

Additionally, cosmological considerations place a limit on the total mass of the neutrinos [14], along with limits from tritium beta decay and neutrinoless double beta decay on the electron neutrino [1, 2]. These experimental results constrain the total mass of the light neutrinos to be less than or of the order of $1 \mathrm{eV}$. Our discussion does not depend on the exact number since the masses are degenerate in this limit. The bound will only become important to our analysis if it approaches the atmospheric mass splitting.

The mixing is characterized by the PMNS matrix, a unitary matrix parameterized by three angles and three phases,

$$
\begin{aligned}
V_{\mathrm{PMNS}} & \equiv L_{e}^{\dagger} L_{\nu} \\
& =\left(\begin{array}{ccc}
c_{12} c_{13} & s_{12} c_{13} & s_{13} e^{-i \delta} \\
-s_{12} c_{23}-c_{12} s_{23} s_{13} e^{i \delta} & c_{12} c_{23}-s_{12} s_{23} s_{13} e^{i \delta} & s_{23} c_{13} \\
s_{12} s_{23}-c_{12} c_{23} s_{13} e^{i \delta} & -c_{12} s_{23}-s_{12} c_{23} s_{13} e^{i \delta} & c_{23} c_{13}
\end{array}\right) \times \operatorname{diag}\left(e^{i \alpha_{1} / 2}, e^{i \alpha_{2} / 2}, 1\right) .
\end{aligned}
$$


For concreteness, we will assume the tribimaximal solution which sets the mixing angles $\theta_{12}=30^{\circ}, \theta_{13}=0^{\circ}, \theta_{23}=45^{\circ}$ [15],

$$
V_{\mathrm{PMNS}}=\left(\begin{array}{ccc}
\sqrt{\frac{2}{3}} & \sqrt{\frac{1}{3}} & 0 \\
-\sqrt{\frac{1}{6}} & \sqrt{\frac{1}{3}} & \sqrt{\frac{1}{2}} \\
\sqrt{\frac{1}{6}} & -\sqrt{\frac{1}{3}} & \sqrt{\frac{1}{2}}
\end{array}\right)
$$

neglecting phases. This is in some sense an extreme solution consistent with the data. Given the several seemingly disparate factors which influence the angles, it seems highly unlikely that any model will predict exactly zero for $\theta_{13}$, or exactly maximal atmospheric mixing, unless carefully designed to do so [6]. Therefore it may well be that experiments eventually favor a less striking set of angles. Furthermore, in a detailed model one would also need to carefully consider renormalization, which can have a significant effect on the mixing angles and mass splittings [16]. [2] We do not address these effects in further detail in this paper because they make little difference in our analysis. We are only looking at relative orders of magnitude of masses and mixing angles. Due to its simple structure, we will use the tribimaximal solution as an experimental input. The critical facts we need are the existence of two large neutrino mixing angles and a relatively weak neutrino mass hierarchy, both of which will remain true despite renormalization effects.

We will assume for now that the tribimaximal structure is generated essentially in the neutrino sector; given the charged lepton hierarchy, we usually expect relatively small rotations in $L_{e}$ compared to the large PMNS entries. Since we are only concerned with orders of magnitude, we will (for now) neglect the charged lepton component. As with the geometric hierarchy discussed in Section 2, there is one exception to this rule associated with a highly asymmetric structure, this time in the charged lepton matrix. Such a lopsided matrix can introduce large rotations, as shown in the Albright-Barr model [7]. This case will be discussed further in Section 8.

The neutrino mass matrix will be diagonalized by the tribimaximal rotations if it has the form

$$
M_{\nu}=V_{\mathrm{PMNS}} D_{\nu} V_{\mathrm{PMNS}}^{\dagger} \propto\left(\begin{array}{ccc}
\left(m_{1}+\frac{1}{2} m_{2}\right) & -\frac{1}{2}\left(m_{1}-m_{2}\right) & \frac{1}{2}\left(m_{1}-m_{2}\right) \\
-\frac{1}{2}\left(m_{1}-m_{2}\right) & \frac{1}{2}\left(\frac{1}{2} m_{1}+m_{2}+\frac{3}{2} m_{3}\right) & -\frac{1}{2}\left(\frac{1}{2} m_{1}+m_{2}-\frac{3}{2} m_{3}\right) \\
\frac{1}{2}\left(m_{1}-m_{2}\right) & -\frac{1}{2}\left(\frac{1}{2} m_{1}+m_{2}-\frac{3}{2} m_{3}\right) & \frac{1}{2}\left(\frac{1}{2} m_{1}+m_{2}+\frac{3}{2} m_{3}\right)
\end{array}\right),
$$

i.e., $L_{\nu}=R_{\nu}=V_{\mathrm{PMNS}}$. The $m$ 's are the physical neutrino masses with an arbitrary phase for $m_{1}$ and $m_{2}$. Since we know the two mass squared differences, we may rewrite these in terms of a single mass,

$$
m_{1}=e^{i \phi_{1}}\left|m_{1}\right|, \quad m_{2}=e^{i \phi_{2}} \sqrt{\left|m_{1}\right|^{2}+\Delta_{\mathrm{sol}}^{2}}, \quad m_{3}=\sqrt{\left|m_{1}\right|^{2}+\Delta_{\mathrm{sol}}^{2} \pm \Delta_{\mathrm{atm}}^{2}}
$$

\footnotetext{
${ }^{2}$ For example, a bimaximal mixing scenario $\left(\theta_{12}, \theta_{23}=45^{\circ}, \theta_{13}=0\right)$ at the GUT scale can produce weak scale mixing angles consistent with the data quoted above [16].
} 
where we have introduced the notation $\Delta \equiv \sqrt{\Delta m^{2}}$. The \pm in the definition of $m_{3}$ represents the choice of normal $(+)$ or inverted ( - ) hierarchy. We take the phase factors $e^{i \phi_{1,2}}$ to be \pm 1 so that there are just a few choices of relative positive or negative to make. Since we are only concerned with orders of magnitude and this will give the extrema, this should not limit the analysis. Then it is simple to scan through the allowed range of $m_{1}$. By doing this, one can observe the patterns of relative order in the neutrino entries which are consistent with experiment. The potentially interesting possibilities are

1. $M_{\nu} \sim\left(\begin{array}{lll}\lambda & \lambda & \lambda \\ \lambda & 1 & 1 \\ \lambda & 1 & 1\end{array}\right)$, corresponding to $m_{1} \ll m_{2} \simeq \Delta_{\text {sol }}$, normal hierarchy.

2. $M_{\nu} \sim\left(\begin{array}{lll}0 & \lambda & \lambda \\ \lambda & 1 & 1 \\ \lambda & 1 & 1\end{array}\right)$, corresponding to $2 m_{1} \simeq m_{2} \simeq \frac{2}{\sqrt{3}} \Delta_{\text {sol }}, \phi_{2}-\phi_{1}=\pi$, normal hierarchy.

3. $M_{\nu} \sim\left(\begin{array}{lll}1 & 0 & 0 \\ 0 & 1 & 1 \\ 0 & 1 & 1\end{array}\right)$, corresponding to $\Delta_{\text {sol }}\left(\Delta_{\text {atm }}\right) \lesssim m_{1} \simeq m_{2} \lesssim \Delta_{\text {atm }}\left(\sqrt{2} \Delta_{\text {atm }}\right), \phi_{2}-\phi_{1}=0$, normal (inverted) hierarchy.

4. $M_{\nu} \sim\left(\begin{array}{lll}1 & 0 & 0 \\ 0 & 1 & 0 \\ 0 & 0 & 1\end{array}\right)$, corresponding to degenerate masses, $\phi_{2}=0, \phi_{1}=0$.

5. $M_{\nu} \sim\left(\begin{array}{lll}1 & 0 & 0 \\ 0 & 0 & 1 \\ 0 & 1 & 0\end{array}\right)$, corresponding to degenerate masses, $\phi_{2}=\pi, \phi_{1}=\pi$.

6. $M_{\nu} \sim\left(\begin{array}{lll}1 & 1 & 1 \\ 1 & 1 & 1 \\ 1 & 1 & 1\end{array}\right)$, corresponding to degenerate masses, $\phi_{2}-\phi_{1}=\pi$.

Here $\lambda \equiv \frac{\Delta_{\text {sol }}}{\Delta_{\text {atm }}} \simeq 0.2$ and 0 should be read as at least a few orders of magnitude smaller than 1 . Any other possibilities should be roughly an interpolation between those listed and we do not expect them to lead to significant deviations from the results following.

The cases with non-degenerate masses, namely the first through third above, violate the geometrical hierarchy naturalness limit discussed in Section 2. In each case the democratic 2-3 block generically leads to two large eigenvalues of order 1 and one large mixing angle. Then the couplings of the first generation give a naive estimate for the third eigenvalue of $\lambda, \lambda^{2}$, and 1 for the first, second, and third cases, respectively. This is not compatible with the eigenvalue ranges listed above, so some unexpected cancellations would have to take place. Moreover, these cases are more compatible with a small $\theta_{12}$ due to the smallness of all off-diagonal first generation entries. The fourth and fifth cases naturally lead to degenerate eigenvalues as listed but imply unnatural precision to account for the large mixing angles.

In short, hierarchical neutrino masses are unexpected in conjunction with large mixing angles, and large mixing angles naturally proceed from large off-diagonal entries in the effective mass matrix. Thus, case 6 above is the most natural simple assumption to account for the experimental data; it is known as a democratic mass matrix [17].

We note that for an inverted hierarchy with $m_{2} \simeq \frac{3}{2 \sqrt{2}} \Delta_{\text {atm }}$, we could have

$$
M_{\nu} \sim\left(\begin{array}{lll}
1 & 1 & 1 \\
1 & 0 & 1 \\
1 & 1 & 0
\end{array}\right), \quad\left(\begin{array}{lll}
1 & 1 & 1 \\
1 & 1 & 0 \\
1 & 0 & 1
\end{array}\right),
$$


depending on the phases $\phi_{1,2}$. These should be thought of as special subcases of case 6 . As will be shown in the next section, these possibilities will only add additional modelling constraints compared to case 6 without additional explanatory power, so they are not particularly interesting in this context. Bearing these caveats in mind we shall, however, consider some cases besides 6 because they may relax other naturalness constraints.

\section{Modelling}

Now we will do a little rearranging of the seesaw formula in terms of the eigenvalues and unitary matrix decomposition of $M_{D}$ :

$$
R_{D}^{\dagger} M_{R}^{-1} R_{D}^{*}=D_{D}^{-1} L_{D}^{\dagger} M_{\nu} L_{D}^{*} D_{D}^{-1}
$$

Applying this to the sixth and henceforth canonical case above, we get

$$
R_{D}^{\dagger} M_{R}^{-1} R_{D}^{*} \sim\left(\begin{array}{ccc}
\frac{1}{\eta^{2}} & \frac{1}{\eta \epsilon} & \frac{1}{\eta} \\
\frac{1}{\eta \epsilon} & \frac{1}{\epsilon^{2}} & \frac{1}{\epsilon} \\
\frac{1}{\eta} & \frac{1}{\epsilon} & 1
\end{array}\right)
$$

where we have kept only the leading terms. The salient point is that, with the assumption $\mu^{\prime}, \nu^{\prime}, \rho^{\prime} \leq 1$, the $L_{D}$ rotations (and similarly the charged lepton rotations) cannot change the orders of the entries. From this we see the apparent double hierarchy for $M_{R}$ : its eigenvalues naturally scale as $\eta^{2}, \epsilon^{2}, 1$ compared to $\eta, \epsilon, 1$ for $M_{D}$.

Most of the other cases are similar and retain at least a $\frac{1}{\eta^{2}}$ ratio between the first and third eigenvalues. For the cases where $M_{\nu}$ has entries less than order one, the unitary rotations can contribute significantly, in particular they can "fill in" the zero entries, but they cannot make any entries larger than order unity in $L_{D}^{\dagger} M_{\nu} L_{D}^{*}$.

There are two cases which may differ importantly from the others. Case 1 in Section 3 is interesting since it yields

$$
R_{D}^{\dagger} M_{R}^{-1} R_{D}^{*} \sim\left(\begin{array}{ccc}
\frac{\lambda}{\eta^{2}} & \frac{1}{\eta \epsilon}\left(\lambda+\mu^{\prime} \frac{\eta}{\epsilon}\right) & \frac{1}{\eta}\left(\lambda+\mu^{\prime} \frac{\eta}{\epsilon}\right) \\
\frac{1}{\eta \epsilon}\left(\lambda+\mu^{\prime} \frac{\eta}{\epsilon}\right) & \frac{1}{\epsilon^{2}} & \frac{1}{\epsilon} \\
\frac{1}{\eta}\left(\lambda+\mu^{\prime} \frac{\eta}{\epsilon}\right) & \frac{1}{\epsilon} & 1
\end{array}\right)
$$

Similarly, for the second case we get

$$
R_{D}^{\dagger} M_{R}^{-1} R_{D}^{*} \sim\left(\begin{array}{ccc}
\frac{1}{\eta^{3 / 2}}\left(\lambda+\mu^{\prime} \frac{\eta}{\epsilon}\right)\left(\frac{\mu^{\prime}}{\sqrt{\epsilon}}+\nu^{\prime}\right) & \frac{1}{\eta \epsilon}\left(\lambda+\mu^{\prime} \frac{\eta}{\epsilon}\right) & \frac{1}{\eta}\left(\lambda+\mu^{\prime} \frac{\eta}{\epsilon}\right) \\
\frac{1}{\eta \epsilon}\left(\lambda+\mu^{\prime} \frac{\eta}{\epsilon}\right) & \frac{1}{\epsilon^{2}} & \frac{1}{\epsilon} \\
\frac{1}{\eta}\left(\lambda+\mu^{\prime} \frac{\eta}{\epsilon}\right) & \frac{1}{\epsilon} & 1
\end{array}\right)
$$

In these cases we see that we have mitigated the largest ratio of entries from $\frac{1}{\eta^{2}}$ to a smaller value, although said ratio remains significantly larger than $\frac{1}{\eta}$. 
Let us now consider the effects of the matrix $R_{D}$ on the canonical case. We will show that, under the current assumptions, one can put additional constraints on $\mu, \nu$ and $\rho$. To begin, we parameterize the inverse heavy neutrino matrix

$$
M_{R}^{-1} \equiv\left(\begin{array}{ccc}
A & B & C \\
B & D & E \\
C & E & F
\end{array}\right)
$$

and evaluate both Eq. (12) and

$$
M_{R}^{-1}=R_{D} D_{D}^{-1} L_{D}^{\dagger} M_{\nu} L_{D}^{*} D_{D}^{-1} R_{D}^{T}
$$

which is just another rearrangement of the seesaw formula. Keeping only potentially leading terms, we find

$$
\begin{aligned}
& A \simeq \frac{1}{\eta^{2}} \\
& B \simeq \frac{\mu}{\eta^{3 / 2} \epsilon^{1 / 2}}+\frac{1}{\eta \epsilon} \\
& C \simeq \frac{\nu}{\eta^{3 / 2}}+\frac{\rho}{\eta \epsilon^{1 / 2}}+\frac{\mu \nu}{\epsilon^{3 / 2}}+\frac{1}{\eta} \\
& D \simeq \frac{\mu^{2}}{\eta \epsilon}+\frac{2 \mu}{\eta^{1 / 2} \epsilon^{3 / 2}}+\frac{2 \rho \mu}{\eta^{1 / 2}}+\frac{1}{\epsilon^{2}} \\
& E \simeq \frac{\mu \nu}{\eta \epsilon^{1 / 2}}+\frac{\nu+\rho \mu}{\eta^{1 / 2} \epsilon}+\frac{\mu}{\eta^{1 / 2} \epsilon^{1 / 2}}+\frac{\rho \nu \epsilon^{1 / 2}}{\eta^{1 / 2}}+\frac{\rho}{\epsilon^{3 / 2}}+\frac{1}{\epsilon} \\
& F \simeq \frac{\nu^{2}}{\eta}+\frac{2 \rho \nu}{\eta^{1 / 2} \epsilon^{1 / 2}}+\frac{2 \nu}{\eta^{1 / 2}}+\frac{\rho^{2}}{\epsilon}+\frac{\rho}{\epsilon^{1 / 2}}+1
\end{aligned}
$$

Now, with a little consideration, one can see that each entry should only be as big as the rightmost term. This is because Eq. (12) must still be satisfied looking only at the order of the terms. For example, we can look at the equation for the (12) entry of Eq. (13) in terms of $A$ through $F$ and $\mu, \nu$, and $\rho$ via Eqs. (3) and (16). This comes out to be

$$
A \mu \sqrt{\frac{\eta}{\epsilon}}+B+C\left(\rho \sqrt{\epsilon}+\mu \nu \frac{\eta}{\sqrt{\epsilon}}\right)+D \mu \sqrt{\frac{\eta}{\epsilon}}+E(\mu \rho+\nu) \sqrt{\eta}+F \rho \nu \sqrt{\eta \epsilon} \sim \frac{1}{\eta \epsilon} .
$$

$B$ appears in this equation with a coeffecient of order 1 , thus any solution to the set of conditions in Eqs. (18) with $B>\frac{1}{\eta \epsilon}$ will apparently not satisfy Eq. (19) $3^{3}$ This is a naturalness condition. One can, of course, numerically satisfy both equations but it requires a cancellation between two

\footnotetext{
${ }^{3}$ Here the important number is actually the ratio $B / F \sim 1 /(\eta \epsilon)$. Using the conventions above we find $F \sim 1$, but there is an overall numerical factor which we omit because it can be absorbed into the dimensionful vevs.
} 
terms to at least an order of magnitude. If we want to avoid the need for a symmetry precisely relating various parameters, the only natural solution is to set $B \sim \frac{1}{\eta \epsilon}$.

Applying the same analysis to the rest of Eqs. (18), we come to the conclusion that

$$
M_{R}^{-1} \sim D_{D}^{-1} L_{D}^{\dagger} M_{\nu} L_{D}^{*} D_{D}^{-1}
$$

or that the hierarchy of $M_{R}^{-1}$ could be even stronger, regardless of $R_{D}$. Then we must impose constraints on the mixing parameters in Eqs. (18) so that the parameters $B-F$ do not become too large:

$$
\mu \lesssim \sqrt{\frac{\eta}{\epsilon}}, \quad \nu \lesssim \sqrt{\eta}, \quad \rho \lesssim \sqrt{\epsilon}
$$

For $\eta \sim 10^{-4}$ and $\epsilon \sim 10^{-2}$, this corresponds to $\mu, \rho \lesssim 10^{-1}$ and $\nu \lesssim 10^{-2}$.

If we take the minimum required suppression and apply it to $\mu^{\prime}, \nu^{\prime}$, and $\rho^{\prime}$ as well, we get the cascade hierarchy pattern 10, 18] for the Dirac matrix,

$$
M_{D} \sim\left(\begin{array}{lll}
\eta & \eta & \eta \\
\eta & \epsilon & \epsilon \\
\eta & \epsilon & 1
\end{array}\right)
$$

For any hierarchical texture of $R_{D}^{\dagger} M_{R}^{-1} R_{D}^{*}$ we will find that $M_{R}^{-1}$ generally retains the same hierarchy. Intuitively, this is because $R_{D}$ will tend to smear out any hierarchy in $M_{R}^{-1}$; the larger entries will be rotated into the smaller. The hierarchy would only be sharpened if there were a very precise relation between $R_{D}$ and $M_{R}^{-1}$, which we have no reason to expect. So in general, if $R_{D}^{\dagger} M_{R}^{-1} R_{D}^{*}$ has a hierarchy of entries, $M_{R}^{-1}$ should have at least as strong a hierarchy. Conversely, to maintain a strong hierarchy in $R_{D}^{\dagger} M_{R}^{-1} R_{D}^{*}$, the unitary rotations cannot be too far from diagonal, a fact reflected in the constraints on $\mu, \nu$ and $\rho$.

For the other possible textures of $M_{\nu}$ with one or two suppressed entries, we mostly find equal or stronger constraints on the $\mu, \nu$, and $\rho$. For example, if the (23) and (32) entries of $M_{\nu}$ are small so that the corresponding entries in $D_{D}^{-1} L_{D}^{\dagger} M_{\nu} L_{D}^{*} D_{D}^{-1}$ are much less than $\frac{1}{\epsilon}$, then we also require $E \ll \frac{1}{\epsilon}$. This in turn imposes stronger constraints on the mixing parameters. This is the situation for cases 3-5 as well as the special sub-cases of 6 mentioned in Section 3 .

It is interesting that the constraints on $\mu, \nu$, and $\rho$ remain valid even if we take the first case of the list,

$$
M_{\nu} \sim\left(\begin{array}{ccc}
\lambda & \lambda & \lambda \\
\lambda & 1 & 1 \\
\lambda & 1 & 1
\end{array}\right), \quad R^{\dagger} M_{R}^{-1} R^{*} \sim\left(\begin{array}{ccc}
\frac{\lambda}{\eta^{2}} & \frac{\lambda}{\eta \epsilon} & \frac{\lambda}{\eta} \\
\frac{\lambda}{\eta \epsilon} & \frac{1}{\epsilon^{2}} & \frac{1}{\epsilon} \\
\frac{\lambda}{\eta} & \frac{1}{\epsilon} & 1
\end{array}\right)
$$

\footnotetext{
${ }^{4}$ Technically, it could be smaller since Eq. (12) depends on experimental numbers. Thus in Eq. (19), it may cancel the theoretical parameter term $\mu /\left(\eta^{3 / 2} \sqrt{\epsilon}\right)$ without fine tuning as long as it is consistent with the experimentally allowed range. At any rate, it would only make the hierarchy stronger since $A \sim \frac{1}{\eta^{2}}$ regardless.
} 
This is because we retain the strong hierarchy along the first column and row, as well as in the (23)-block, whose entries remain less than or equal in order to the first generation entries.

The one exceptional case is the other form noted before, case 2. This leads one to the conclusion

$$
M_{R}^{-1} \sim\left(\begin{array}{ccc}
\frac{1}{\eta^{3 / 2}}\left(\lambda+\mu^{\prime} \frac{\eta}{\epsilon}\right)\left(\frac{\mu^{\prime}}{\sqrt{\epsilon}}+\nu^{\prime}\right) & \frac{1}{\eta \epsilon}\left(\lambda+\mu^{\prime} \sqrt{\frac{\eta}{\epsilon}}\right) & \frac{1}{\eta}\left(\lambda+\mu^{\prime} \sqrt{\frac{\eta}{\epsilon}}\right) \\
\frac{1}{\eta \epsilon}\left(\lambda+\mu^{\prime} \sqrt{\frac{\eta}{\epsilon}}\right) & \frac{1}{\epsilon^{2}} & \frac{1}{\epsilon} \\
\frac{1}{\eta}\left(\lambda+\mu^{\prime} \sqrt{\frac{\eta}{\epsilon}}\right) & \frac{1}{\epsilon} & 1
\end{array}\right)
$$

and the naturalness conditions

$$
\mu \lesssim \frac{\sqrt{\eta}}{\sqrt{\epsilon} \lambda} \sim 1, \quad \nu \lesssim \frac{\sqrt{\eta}}{\lambda} \sim 0.1, \quad \rho \leq \sqrt{\epsilon} \sim 0.1
$$

So in this case we are not as constrained as the cascade pattern but still more constrained than the geometric pattern; only the constraint on $\rho$ remains the same. This makes sense since, in this case, we have a relatively weak hierarchy in the first row and column compared to the canonical case. Therefore, we find weaker constraints on the rotation parameters for the first generation.

In general then, we are led to both a double (or at least enhanced) hierarchy for $M_{R}$ and a cascade (or sub-geometrical) pattern for $M_{D}$ in a simple type-I scenario.

\section{CKM Constraints}

The Dirac mass matrices of quarks and leptons are related by $\mathrm{SO}(10)$ and possibly family symmetries. Thus, we should also consider the size of the unitary rotations in the up and down quark mass matrices, which are measurable through the CKM matrix, $V_{\mathrm{CKM}} \equiv L_{u}^{\dagger} L_{d}$. The experimental CKM values are [1]

$$
V_{\mathrm{CKM}}=\left(\begin{array}{ccc}
1 & 0.226 \pm 0.002 & {[4.3 \pm 0.3] \times 10^{-3}} \\
0.23 \pm 0.01 & 1 & {[4.2 \pm 0.06] \times 10^{-2}} \\
{[7.4 \pm 0.8] \times 10^{-3}} & 3.5 \times 10^{-2} & 1
\end{array}\right)
$$

If we suppose for the moment a geometric pattern for both the up and down quark matrices, then the predicted CKM matrix is

$$
\begin{aligned}
V_{\mathrm{CKM}} \simeq & \left(\begin{array}{ccc}
1 & \sqrt{\frac{m_{d}}{m_{s}}}-\sqrt{\frac{m_{u}}{m_{c}}}+\sqrt{\frac{m_{u} m_{s}}{m_{t} m_{b}}} & \sqrt{\frac{m_{d}}{m_{b}}}-\sqrt{\frac{m_{u}}{m_{t}}}-\sqrt{\frac{m_{u} m_{s}}{m_{c} m_{b}}} \\
-\sqrt{\frac{m_{d}}{m_{s}}}+\sqrt{\frac{m_{u}}{m_{c}}}-\sqrt{\frac{m_{c} m_{d}}{m_{t} m_{b}}} & 1 & \sqrt{\frac{m_{s}}{m_{b}}}-\sqrt{\frac{m_{c}}{m_{t}}}+\sqrt{\frac{m_{u} m_{d}}{m_{c} m_{b}}} \\
-\sqrt{\frac{m_{d}}{m_{b}}}+\sqrt{\frac{m_{u}}{m_{t}}}-\sqrt{\frac{m_{c} m_{d}}{m_{t} m_{s}}} & -\sqrt{\frac{m_{s}}{m_{b}}}+\sqrt{\frac{m_{c}}{m_{t}}}-\sqrt{\frac{m_{u} m_{d}}{m_{t} m_{s}}} & 1
\end{array}\right) \\
& \simeq\left(\begin{array}{ccc}
1 & 0.23-0.06+4 \times 10^{-4} & 0.03-0.003-0.008 \\
-0.23+0.06-0.001 & 1 & 0.14-0.04+0.002 \\
-0.03+0.003-0.01 & -0.14+0.04-7 \times 10^{-4} & 1
\end{array}\right) .
\end{aligned}
$$


A few features are striking. One is that the geometric ratio $\sqrt{\frac{m_{d}}{m_{s}}}-\sqrt{\frac{m_{u}}{m_{c}}}$ nicely reproduces the experimental value for the first-second generation mixing [20]. The dominant term comes from the down quark mixing, while the contribution from the up quark mixing is significantly too small to account for the mixing by itself. Secondly, the down quark contribution to the firstthird mixing is too large by roughly an order of magnitude. Lastly, the down quark contribution to the second-third generation mixing is also too large by roughly a factor of three. So the geometric hierachy does a good job for the Cabibbo angle but gives too much mixing with the third generation.

This result is consistent with a partially cascade structure in $M_{d}$ and $M_{u}$. The relatively large Cabibbo angle indicates that the down quark matrix should be close to geometrical in the 1-2 block. However, it will fit the data better if it is cascade-like in the third generation. If the same were true of $M_{D}$, we would be consistent with the second case from Section 3. On the other hand, since $1 \mathbf{6}_{i} \mathbf{1 6} \mathbf{1 6}_{H} \mathbf{1 6}_{H}$ only contributes to the down quark and charged lepton matrices, the neutrino matrix could remain completely cascade-like without conflict.

\section{Implementing the Cascade Hierarchy}

Since we argue that a cascade texture is theoretically desirable, we will investigate how it can be generated. We will make use of the Froggatt-Nielsen mechanism [11] and consider a global $U(1) \times \mathbb{Z}_{2} \times \mathbb{Z}_{2}^{\prime}$ symmetry. We introduce three $\mathrm{SO}(10)$ singlets $\phi_{i}$. The flavor symmetry is broken spontaneously at a high scale $m$ by vevs of the singlet fields, which we expect to be all of the same order, $\langle\phi\rangle$. The symmetry breaking is assumed to be transmitted to quarks and leptons through interactions with heavy particles so that the Yukawa couplings are constructed out of powers of $\zeta \equiv\langle\phi\rangle / m$ with a texture dictated by the family symmetry.

We assign the following charges:

\begin{tabular}{|c||c|c|c|c||c|c|c|}
\hline Field & $\mathbf{1 6}_{1}$ & $\mathbf{1 6}_{2}$ & $\mathbf{1 6}_{3}$ & $\mathbf{1 0}_{H}$ & $\phi_{1}$ & $\phi_{2}$ & $\phi_{3}$ \\
\hline \hline $\mathrm{U}(1)$ & 2 & 1 & 0 & 0 & -1 & 0 & 0 \\
\hline $\mathbb{Z}_{2}$ & - & - & + & + & + & - & + \\
\hline $\mathbb{Z}_{2}^{\prime}$ & - & + & + & + & + & + & - \\
\hline
\end{tabular}

Then the operator $M_{10}^{i j} \mathbf{1 6}_{i} \mathbf{1 6}_{j} \mathbf{1 0}_{H}$ originates from $\Phi^{i j} \mathbf{1 6}_{i} \mathbf{1 6}_{j} \mathbf{1 0}_{H}$, where $\Phi$ represents the higherdimensional couplings,

$$
\Phi=\left(\begin{array}{ccc}
\frac{1}{m^{4}}\left(\phi_{1}\right)^{4} & \frac{1}{m^{4}}\left(\phi_{1}\right)^{3} \phi_{3} & \frac{1}{m^{4}}\left(\phi_{1}\right)^{2} \phi_{2} \phi_{3} \\
\frac{1}{m^{4}}\left(\phi_{1}\right)^{3} \phi_{3} & \frac{1}{m^{2}}\left(\phi_{1}\right)^{2} & \frac{1}{m^{2}} \phi_{1} \phi_{2} \\
\frac{1}{m^{4}}\left(\phi_{1}\right)^{2} \phi_{2} \phi_{3} & \frac{1}{m^{2}} \phi_{1} \phi_{2} & 1
\end{array}\right),
$$

\footnotetext{
${ }^{5}$ Since the largest terms come from the down quark sector, the CKM values are also consistent with a geometric hierarchy in $M_{u}$. Given $\mathrm{SO}(10)$ relations and possible family symmetries, the simplest assumption is that $M_{u}$ has a similar hierarchy structure to $M_{d}$.
} 
so that

$$
M_{10} \sim\left(\begin{array}{ccc}
\zeta^{4} & \zeta^{4} & \zeta^{4} \\
\zeta^{4} & \zeta^{2} & \zeta^{2} \\
\zeta^{4} & \zeta^{2} & 1
\end{array}\right)
$$

This is the cascade form of Eq. (22) with $\eta=\zeta^{4}$ and $\epsilon=\zeta^{2}$. The same pattern can easily be reproduced in the other operators which contribute to fermion masses. Note that in the absence of the $\mathbb{Z}_{2}$ symmetries we would have generated a geometric hierarchy.

We must also consider whether a cascade hierarchy can naturally accomodate the fermion masses in a unified theory. Restricting ourselves to two generations, the operators discussed in Section 2 contribute to the (normalized) mass matrices as follows:

$$
\begin{array}{ll}
M_{u}=\left(\begin{array}{cc}
\alpha^{\prime} & \alpha+\beta \\
\alpha-\beta & 1
\end{array}\right), & M_{D}=\left(\begin{array}{cc}
\alpha^{\prime} & \alpha-3 \beta \\
\alpha+3 \beta & 1
\end{array}\right), \\
M_{d}=\left(\begin{array}{cc}
\alpha^{\prime}+\gamma^{\prime} & \alpha+\beta+\gamma \\
\alpha-\beta+\gamma & 1
\end{array}\right), & M_{e}=\left(\begin{array}{cc}
\alpha^{\prime}+\gamma^{\prime} & \alpha-3 \beta+\gamma \\
\alpha+3 \beta+\gamma & 1
\end{array}\right) .
\end{array}
$$

Here, the terms $\alpha$ and $\alpha^{\prime}$ parameterize the operator $1 \mathbf{1 6}_{i} \mathbf{1 6}_{j} \mathbf{1 0}_{H}$. The parameter $\beta$ derives from $16_{i} 16_{j} 10_{H} 45_{H}$, while $\gamma$ and $\gamma^{\prime}$ characterize $16_{i} 16_{j} 16_{H} 16_{H}^{\prime}$. Looking at the determinants, we calculate the mass ratios:

$$
\begin{aligned}
\frac{m_{c}}{m_{t}} & \simeq\left|\alpha^{\prime}-\alpha^{2}+\beta^{2}\right|, & \epsilon & \simeq\left|\alpha^{\prime}-\alpha^{2}+9 \beta^{2}\right|, \\
\frac{m_{s}}{m_{b}} & \simeq\left|\alpha^{\prime}+\gamma^{\prime}+\beta^{2}-(\alpha+\gamma)^{2}\right|, & \frac{m_{\mu}}{m_{\tau}} & \simeq\left|\alpha^{\prime}+\gamma^{\prime}+9 \beta^{2}-(\alpha+\gamma)^{2}\right| .
\end{aligned}
$$

As expected, $\beta$ accounts for the difference of down quark and charged fermion masses,

$$
8 \beta^{2}=\frac{m_{\mu}}{m_{\tau}} \mp \frac{m_{s}}{m_{b}} \simeq\left\{\begin{array}{l}
4 \times 10^{-2}- \\
8 \times 10^{-2}+
\end{array}\right.
$$

where we used $\left(m_{\mu} / m_{\tau}\right)_{\mathrm{GUT}} \simeq 0.06$ and $\left(m_{s} / m_{b}\right)_{\mathrm{GUT}} \simeq 0.02$. Since we wish to minimize offdiagonal terms in a cascade-like matrix, we will use the smaller value for $\beta$,

$$
\beta \simeq 7 \times 10^{-2} .
$$

Then we obtain

$$
\epsilon=\frac{m_{c}}{m_{t}}+8 \beta^{2} \simeq 7 \times 10^{-2},
$$

with $\left(m_{c} / m_{t}\right)_{\mathrm{GUT}} \simeq 0.03$.

\footnotetext{
${ }^{6}$ The larger value, $\beta \simeq 0.1$, leads to $\epsilon \simeq 0.01$.
} 
In order to have a cascade form for $M_{D}$, we require $\alpha^{\prime} \sim \alpha \pm 3 \beta \sim \epsilon$. Since $3 \beta \simeq 0.2$, this implies $\alpha^{\prime} \sim 0.1$, independent of $\alpha$. This value of $\alpha^{\prime}$ can be consistent with the value of $\epsilon$ in Eq. (34), but it needs to cancel significantly with $\alpha^{2}$ to ensure a suitably small value for $m_{c} / m_{t}$. Conversely, $m_{c} / m_{t}$ implies $\alpha^{\prime} \lesssim 10^{-2}$, which leads to a geometric hierarchy in $M_{D}$. Since we have been trying to avoid requiring the cancellation of theoretical parameters, this simple cascade ansatz is problematic.

One particularly attractive way out of this dilemma is to consider the possibility that $M_{D}$, but not $M_{u}$, receives additional contributions, e.g., via particular higher-dimensional operators. If such an operator gave a contribution to the (22)-element of $M_{D}$ of order $\epsilon \sim 0.1, \alpha^{\prime}$ could be made sufficiently small. We consider such a scenario in the following section.

\section{New Contributions to $M_{D}$}

In Section 4 we saw that the observed pattern of neutrino masses and mixings leads us to an enhanced hierarchy for $M_{R}$, compared to $M_{D}$. One should note, however, that while $M_{D}$ is related to the observed quark and charged lepton hierarchies by $\mathrm{SO}(10)$ and any family symmetries, it is not directly observed. In particular, one may include another operator, $\mathbf{1 6}_{i} \mathbf{1 6}_{j} \overline{\mathbf{1 6}}_{H} \overline{\mathbf{1 6}}_{H}^{\prime}$. As noted above, the weak doublet in $\overline{\mathbf{1 6}}_{H}^{\prime}$ can acquire a weak scale vev $u^{\prime}$ such that this operator potentially contributes to the up quark and neutrino masses. However, it can be constructed to contribute only to the Dirac neutrino matrix. In this case we expect $u^{\prime}<u$, since $u$ is required to generate a large top quark mass and the sum of the squares of weak scale vevs must equal $(246 \mathrm{GeV})^{2}$.

A simple possibility for generating this operator is to integrate out $\mathrm{SO}(10)$ singlets, $\mathbf{S}$, at some scale above the relevant GUT scale vevs. For this purpose we can propose the operators

$$
\bar{M}_{i j} \mathbf{1 6}_{i} \overline{\mathbf{1 6}}_{H} \mathbf{S}_{j}+\bar{M}_{i j}^{\prime} \mathbf{1 6} \overline{\mathbf{1 6}}_{H}^{\prime} \mathbf{S}_{j}+m_{s}\left(M_{S}\right)_{i j} \mathbf{S}_{i} \mathbf{S}_{j}
$$

We assume at least three singlets to guarantee that all three righthanded neutrinos become heavy. As usual, we define $m_{s}$ to have units of mass so that $M_{S}$ is dimensionless with entries of order 1 or smaller, and similarly we normalize $\bar{M}$ and $\bar{M}^{\prime}$ in Eq. (38). In the following analysis we assume that all the $\mathbf{S}$ singlets are integrated out to generate an effective Majorana mass for the $N$ 's. To compute this via a straightforward seesaw mechanism, we will work in the basis where $M_{S}$ is diagonal and impose the conditions

$$
\left(M_{S}\right)_{i i}>\frac{v}{m_{s}}
$$

for all $i$. 
The mass matrix for the electrically neutral particles reads]

$$
\left(\begin{array}{lll}
\nu & N & S
\end{array}\right)\left(\begin{array}{ccc}
0 & \frac{1}{2} u M_{D} & \frac{1}{2} u^{\prime} \bar{M}^{\prime} \\
\frac{1}{2} u M_{D}^{T} & 0 & \frac{1}{2} v \bar{M} \\
\frac{1}{2} u^{\prime} \bar{M}^{T} & \frac{1}{2} v \bar{M}^{T} & m_{s} M_{S}
\end{array}\right)\left(\begin{array}{c}
\nu \\
N \\
S
\end{array}\right) .
$$

As derived in Appendix $\mathrm{A}$, the light neutrino mass matrix is then given by

$$
M_{\nu} \simeq M_{D}\left(\bar{M}^{-1}\right)^{T} M_{S} \bar{M}^{-1} M_{D}^{T}-\frac{x}{2}\left[\bar{M}^{\prime} \bar{M}^{-1} M_{D}^{T}+M_{D}\left(\bar{M}^{\prime} \bar{M}^{-1}\right)^{T}\right], \quad x \equiv \frac{u^{\prime} v}{u m_{s}} \ll 1 .
$$

The mass of the heaviest neutrino is of order $u^{2} m_{s} / v^{2}$. It is crucial that, in the final formula, $M_{D}$ appears in all terms, i.e., terms quadratic in $\bar{M}^{\prime} \bar{M}^{-1}$ have not appeared.

Let us study the effect of the new contributions. We parametrize the various matrices as follows:

$$
M_{R}^{-1}=\left(\bar{M}^{-1}\right)^{T} M_{S} \bar{M}^{-1} \equiv\left(\begin{array}{ccc}
A & B & C \\
B & D & E \\
C & E & F
\end{array}\right), \quad \bar{M}^{\prime} \bar{M}^{-1}=\left(\begin{array}{ccc}
a & b & c \\
b^{\prime} & d & e \\
c^{\prime} & e^{\prime} & f
\end{array}\right)
$$

(note that the matrix $\bar{M}^{\prime} \bar{M}^{-1}$ is generally not symmetric), and

$$
M_{D}^{-1} M_{\nu}\left(M_{D}^{-1}\right)^{T}=M_{R}^{-1}-\frac{x}{2}\left[M_{D}^{-1} \bar{M}^{\prime} \bar{M}^{-1}+\left(\bar{M}^{\prime} \bar{M}^{-1}\right)^{T}\left(M_{D}^{-1}\right)^{T}\right]=\left(\begin{array}{ccc}
A^{\prime} & B^{\prime} & C^{\prime} \\
B^{\prime} & D^{\prime} & E^{\prime} \\
C^{\prime} & E^{\prime} & F^{\prime}
\end{array}\right)
$$

The last matrix, with primed capital letters, is the total effective matrix which takes the place of $M_{R}^{-1}$ in Section 6 . The unprimed capital letters parameterize the familiar heavy neutrino matrix and the lower case letters parameterize the new terms. Before proceeding to consider the effects of these new terms, we note that $M_{R}$ can easily acquire a double hierarchy if it is generated by integrating out heavy singlets, as described above. If $\bar{M}$ has a hierarchy comparable to $M_{D}$ and $M_{S}$ is roughly democratic, a double hierarchy occurs naturally.

We can write the total effective parameters in terms of these old and new components and perform the same analysis on the total effective matrix $\left(A^{\prime}-F^{\prime}\right)$ as we did on the simple type-I parameters $(A-F)$ in Section 6 . Then we obtain the following set of equations:

$$
\begin{aligned}
& A^{\prime} \simeq A+\left[\frac{a}{\eta}+b^{\prime} \frac{\mu^{\prime}}{\sqrt{\eta \epsilon}}+c^{\prime} \frac{\nu^{\prime}}{\sqrt{\eta}}\right] x \\
& B^{\prime} \simeq B+\frac{1}{2}\left[a \frac{\mu}{\sqrt{\eta \epsilon}}+\frac{b}{\eta}+\frac{b^{\prime}}{\epsilon}+c^{\prime} \frac{\rho^{\prime}}{\sqrt{\epsilon}}+d \frac{\mu^{\prime}}{\sqrt{\eta \epsilon}}+e^{\prime} \frac{\nu^{\prime}}{\sqrt{\eta}}\right] x \\
& C^{\prime} \simeq C+\frac{1}{2}\left[a \frac{\nu}{\sqrt{\eta}}+b^{\prime} \frac{\rho}{\sqrt{\epsilon}}+c^{\prime}+\frac{c}{\eta}+e \frac{\mu^{\prime}}{\sqrt{\eta \epsilon}}+f \frac{\nu^{\prime}}{\sqrt{\eta}}\right] x
\end{aligned}
$$

\footnotetext{
${ }^{7}$ Barr calls this scenario a type-III seesaw mechanism 19; however, it can also be understood as a product of two type-I mechanisms.
} 


$$
\begin{aligned}
& D^{\prime} \simeq D+\left[b \frac{\mu}{\sqrt{\eta \epsilon}}+\frac{d}{\epsilon}+e^{\prime} \frac{\rho^{\prime}}{\sqrt{\epsilon}}\right] x \\
& E^{\prime} \simeq E+\frac{1}{2}\left[b \frac{\nu}{\sqrt{\eta}}+c \frac{\mu}{\sqrt{\eta \epsilon}}+d \frac{\rho}{\sqrt{\epsilon}}+\frac{e}{\epsilon}+e^{\prime}+f \frac{\rho^{\prime}}{\sqrt{\epsilon}}\right] x \\
& F^{\prime} \simeq F+\left[c \frac{\nu}{\sqrt{\eta}}+e \frac{\rho}{\sqrt{\epsilon}}+f\right] x
\end{aligned}
$$

In these equations we have kept only the leading terms. In doing so, we make use of the important fact that the constraints on $\mu, \nu$, and $\rho$ still apply. They follow from consideration of the experimental data and the geometric constraints on $M_{D}$ only.»

Although these equations still appear somewhat complicated, the requirement that we fit the same hierarchy of orders as imposed in Eqs. (18) can only be satisfied in a few ways. In general, the new terms give us new parameters which could play a role in a precision fit to the data, but they will not affect the conclusions of this paper unless they dominate over the old terms. Let us consider the canonical case, which implies

$$
\left(\begin{array}{lll}
A^{\prime} & B^{\prime} & C^{\prime} \\
B^{\prime} & D^{\prime} & E^{\prime} \\
C^{\prime} & E^{\prime} & F^{\prime}
\end{array}\right) \propto\left(\begin{array}{ccc}
\frac{1}{\eta^{2}} & \frac{1}{\eta \epsilon} & \frac{1}{\eta} \\
\frac{1}{\eta \epsilon} & \frac{1}{\epsilon^{2}} & \frac{1}{\epsilon} \\
\frac{1}{\eta} & \frac{1}{\epsilon} & 1
\end{array}\right) .
$$

Examining Eq. (41), this puts some initial constraints on our new parameters. For example,

$$
\frac{b x}{\eta} \leq B^{\prime} \sim \frac{F^{\prime}}{\eta \epsilon} .
$$

These constraints may be summarized in matrix form:

$$
\left(\begin{array}{lll}
a & b & c \\
b^{\prime} & d & e \\
c^{\prime} & e^{\prime} & f
\end{array}\right) \lesssim\left(\begin{array}{ccc}
\frac{1}{\eta} & \frac{1}{\epsilon} & 1 \\
\frac{1}{\eta} & \frac{1}{\epsilon} & 1 \\
\frac{1}{\eta} & \frac{1}{\epsilon} & 1
\end{array}\right) \frac{F^{\prime}}{x} .
$$

Taking these restrictions into account, we conclude that to satisfy $\frac{A^{\prime}}{F^{\prime}} \sim \frac{1}{\eta^{2}}$ we must have

$$
A \sim F^{\prime} \frac{1}{\eta^{2}} \quad \text { or } \quad a \sim F^{\prime} \frac{1}{\eta x} .
$$

The latter case is initially appealing because one can apparently trade the strong double hierarchy constraint on $M_{R}$ for a weaker standard hierarchy in $\bar{M}^{\prime} \bar{M}^{-1}$ if the term involving a dominates.

This turns out not to be feasible. Recall that $\bar{M}$ and $\bar{M}^{\prime}$ have all entries of order 1 or less. Thus, if $a=F^{\prime} \frac{1}{\eta x}$, there exists some $i$ and some $n \geq 1$ for which

$$
\bar{M}_{1 i}^{\prime}=\frac{1}{n} \quad \text { and } \quad \bar{M}_{i 1}^{-1}=\frac{n F^{\prime}}{\eta x}
$$

\footnotetext{
${ }^{8}$ This would not be the case if there were new terms in the effective total matrix which did not involve $M_{D}$.
} 
(cf. Eq. (39)). Then the assumption that the $a$ term dominates over $A$ gives us the inequality

$$
\frac{1}{\eta^{2}} \sim \frac{a x}{\eta F^{\prime}} \geq \frac{A}{F^{\prime}}=\frac{1}{F^{\prime}}\left[\left(\bar{M}^{-1}\right)^{T} M_{S} \bar{M}^{-1}\right]_{11} \geq\left(\frac{n}{\eta x}\right)^{2}\left(M_{S}\right)_{i i} F^{\prime}
$$

from which we obtain $\left(M_{S}\right)_{i i} F^{\prime} \leq \frac{x^{2}}{n^{2}}$. Applying the seesaw constraint on $M_{S}$ and inserting the definition of $x$ gives us

$$
\frac{v}{m_{s}} F^{\prime} \leq\left(M_{S}\right)_{i i} F^{\prime} \leq \frac{u^{\prime 2} v^{2}}{n^{2} u^{2} m_{s}^{2}} .
$$

This requires $F^{\prime}$ to be too small, that is,

$$
1 \lesssim\left[\left(\bar{M}^{-1}\right)^{T} M_{S} \bar{M}^{-1}\right]_{33}=F \leq F^{\prime} \leq \frac{u^{\prime 2} v}{n^{2} u^{2} m_{s}}
$$

Since $v \ll m_{s}$ and $u^{\prime} \lesssim u$, this condition cannot be satisfied. Thus the additional contributions cannot dominate over the type-I contributions or change the need for a double hierarchy.

One can instead look at case 1 from Section 3. If the new terms dominate in the largest ratio, which is still $A^{\prime} / F^{\prime}$, this implies $\bar{M}_{i 1}^{-1}=\lambda \frac{n F^{\prime}}{\eta x}$. Proceeding as in the canonical case above, one finds $\lambda\left(M_{S}\right)_{i i} F^{\prime} \leq \frac{x^{2}}{n^{2}}$. Since we require $F^{\prime} \geq 1$ this is only possible if

$$
x^{2} \geq\left(M_{S}\right)_{i i} \lambda n^{2} \geq \frac{v}{m_{s}} \lambda n^{2},
$$

or equivalently,

$$
\lambda \leq \frac{u^{\prime 2} v}{n^{2} u^{2} m_{s}} \ll 1
$$

This is a very marginal case since we are relying on $v \ll m_{s}$ to use the seesaw formula as a valid approximation and $\lambda \sim 0.2$.

If we proceed nonetheless, then we impose the conditions on $B^{\prime}$ :

$$
\frac{\lambda}{\eta \epsilon} \sim \frac{B^{\prime}}{F^{\prime}} \geq \frac{B}{F^{\prime}} \sim \frac{1}{F^{\prime}} \sum_{k} \bar{M}_{k 1}^{-1} \bar{M}_{k 2}^{-1}
$$

which implies the constraint $\bar{M}_{i 2}^{-1} \leq x /\left[n \epsilon\left(M_{S}\right)_{i i}\right]$. Now we turn to $D^{\prime} \sim \frac{1}{\epsilon^{2}}$. By similar reasoning as in the canonical case it can be shown that $D$ must dominate to satisfy $D^{\prime}$ of the appropriate magnitude, due to the suppression of the new terms by $x$. Then

$$
\frac{1}{\epsilon^{2}} \sim \frac{D^{\prime}}{F^{\prime}} \geq \frac{D}{F^{\prime}} \sim \frac{\left(M_{S}\right)_{j j}}{F^{\prime}}\left(\bar{M}_{j 2}^{-1}\right)^{2}
$$

for some $j \neq i$, which gives us the condition

$$
\bar{M}_{j 2}^{-1} \sim \frac{1}{\epsilon} \sqrt{\frac{F^{\prime}}{\left(M_{S}\right)_{j j}}} .
$$


This in turn implies

$$
\bar{M}_{j 1}^{-1} \leq \frac{\lambda}{\eta} \sqrt{\frac{F^{\prime}}{\left(M_{S}\right)_{j j}}}
$$

so as not to violate the bound on $b$. We find then that the new contributions can technically dominate in the (11) entry but the type-I terms remain comparable and dominate in other entries, still exhibiting a strong hierarchy compared to $M_{D}$.

The related case 2 , with $\left(M_{\nu}\right)_{11} \ll \lambda$, is, not surprisingly, similar. One finds that the new term $a$ can dominate if $\lambda \sqrt{\frac{\eta}{\epsilon}} \leq \frac{u^{\prime 2} v}{n^{2} u^{2} m_{s}}$, which provides somewhat more room for consistency with the seesaw approximation. The constraints on the matrices are

$$
\bar{M}_{i 1}^{-1} \simeq \frac{\lambda n F^{\prime}}{x \sqrt{\eta \epsilon}}, \quad \bar{M}_{i 2}^{-1} \leq \frac{x}{n \sqrt{\eta \epsilon}\left(M_{S}\right)_{i i}}, \quad \bar{M}_{j 2}^{-1} \simeq \frac{1}{\epsilon} \sqrt{\frac{F^{\prime}}{\left(M_{S}\right)_{j j}}}, \quad \bar{M}_{j 1}^{-1} \leq \frac{\lambda}{\eta} \sqrt{\frac{F^{\prime}}{\left(M_{S}\right)_{j j}}} .
$$

In both cases the new terms can dominate in some entries, but the type-I terms remain important and retain a strong, albeit not quite double, hierarchy. We note that this is due largely to the structure of the theory: if $M_{R}$ is a dimension-five operator generated by integrating out singlets, then a hierarchy in $\bar{M}$ similar to that in $M_{D}$ naturally leads to a doubled hierarchy in $M_{R}$. Due to the suppression of the new terms by $v / m_{s}, M_{R}$ will always play an important role.

It is interesting that even with the $\mathbf{1 6}_{i} \mathbf{1 6} \overline{\mathbf{1 6}}_{H} \overline{\mathbf{1 6}}_{H}^{\prime}$ operator only contributing to the neutrino sector, we still derive the cascade constraints. Although this operator only contributes to the Dirac neutrino matrix, the constraints apply to the operators which generate the up quark matrix. This follows from the precise relations between the higher dimensional operators induced by their common origin. These relations result in $M_{D}$ appearing in all terms of the formula for $M_{\nu}$. As a consequence of the persistent cascade constraints, we cannot use the new terms to solve the mass splitting problems discussed in Section 6 .

If one treats $\mathbf{1 6}_{i} \mathbf{1 6} \overline{\mathbf{1 6}}_{H} \overline{\mathbf{1 6}}_{H}^{\prime}$ and $\mathbf{1 6}_{i} \mathbf{1 6}_{j} \overline{\mathbf{1 6}}_{H} \overline{\mathbf{1 6}}_{H}$ as independent, it is possible to relax said constraints. That is, in the discussion above both operators depend on the coupling $\bar{M}_{i j} \mathbf{1 6}_{i} \overline{\mathbf{1 6}}_{H} \mathbf{S}_{j}$ and are therefore related. If we allow them to vary arbitrarily, then the modified seesaw formula in Eq. (38) would have additional terms which did not involve $M_{D}$. In effect, we would be adding new terms to the Dirac neutrino matrix which could strongly alter its hierarchy compared to the quarks and charged leptons. If this resulted in a relatively weak Dirac neutrino hierarchy, $M_{R}$ would have a correspondingly weakened hierarchy and the mixing parameter constraints would also weaken. However, as shown above, this is not necessarily the case when one begins with a more complete theory.

In general, if one can weaken the Dirac neutrino hierarchy without upsetting the charged fermion hierarchies, the requirement of a double hierarchy in $M_{R}$ and a cascade hierarchy in $M_{D}$ becomes less restrictive, since they are specified relative to the eigenvalue hierarchy of $M_{D}$. One possibility for doing so may be to introduce a vector-like fourth generation of down quarks and leptons at the GUT scale. This can relate $M_{D}$ to the down quark hierarchy such that the hierarchy of $M_{R}$ is similar to that of the up quarks [21]. 


\section{Lopsided Models}

Thus far, we have not allowed for any cancellations between terms in our equations, in keeping with our aim to eliminate unnatural models. There are, however, two scenarios where one must be more careful. These are cases where unitary rotations play a very significant role either due to large rotations or small entries in the neutrino matrix.

In this section we will consider the first type of these cases, lopsided models, wherein the operator $\mathbf{1 6}_{i} \mathbf{1 6}_{j} \mathbf{1 6} \mathbf{6}_{H} \mathbf{1 6}_{H}^{\prime}$ is constructed so as to contribute in a highly asymmetrical way to the down quark and charged lepton mass matrices [7].9] These lopsided matrices can yield a natural hierarchy while violating the geometric pattern limit discussed above. Lopsidedness results in large off-diagonal terms in the unitary rotations on one side of the matrix but not both.

To illustrate these features we will restrict ourselves to two generations first. The following table summarizes the three natural cases we have discussed for a generic matrix $M$ with eigenvalues $\epsilon$ and 1 , which is diagonalized by the unitary rotation matrices $L$ and $R$.

\begin{tabular}{|l|c|c|c|}
\hline Hierarchy & $M$ & $L$ & $R$ \\
\hline \hline Geometric & $\left(\begin{array}{cc}\epsilon & \sqrt{\epsilon} \\
\sqrt{\epsilon} & 1\end{array}\right)$ & $\left(\begin{array}{cc}1 & \sqrt{\epsilon} \\
\sqrt{\epsilon} & 1\end{array}\right)$ & $\left(\begin{array}{cc}1 & \sqrt{\epsilon} \\
\sqrt{\epsilon} & 1\end{array}\right)$ \\
\hline Cascade & $\left(\begin{array}{cc}\epsilon & \epsilon \\
\epsilon & 1\end{array}\right)$ & $\left(\begin{array}{ll}1 & \epsilon \\
\epsilon & 1\end{array}\right)$ & $\left(\begin{array}{ll}1 & \epsilon \\
\epsilon & 1\end{array}\right)$ \\
\hline Lopsided & $\left(\begin{array}{ll}\epsilon & \epsilon \\
1 & 1\end{array}\right)$ & $\left(\begin{array}{ll}1 & \epsilon \\
\epsilon & 1\end{array}\right)$ & $\left(\begin{array}{ll}1 & 1 \\
1 & 1\end{array}\right)$ \\
\hline
\end{tabular}

For both the geometric and cascade cases $L$ and $R$ are similar to each other. As expected, the off-diagonal entries of $L$ and $R$ for the cascade case are smaller than in the geometric case.

The lopsided case, being highly asymmetric, leads to very different rotation matrices on the left and right. We see that to generate large mixing on one side, i.e., $R$ with all entries of the same order, we are lead to $L$ being closer to diagonal than in the geometric case. Rather, it is similar to the cascade rotation matrices. So in this simple case, to preserve naturalness, there is a tradeoff between the left and right sides. If one side's unitary rotation violates the geometric naturalness bound, the other's is concomitantly constrained to be closer to unity.

To take potentially large mixing in the charged lepton sector into account, we have to reevaluate our seesaw formula. In Eq. (12), we neglected the rotations from the charged lepton sector, parametrized by $L_{e}$ (cf. Eq. (7)). To include them we rewrite the formula as

$$
R_{D}^{\dagger} M_{R}^{-1} R_{D}^{*}=D_{D}^{-1} V_{0} M_{\nu}^{\prime} V_{0}^{T} D_{D}^{-1},
$$

where

$$
M_{\nu}^{\prime} \equiv V_{\mathrm{PMNS}} D_{\nu} V_{\mathrm{PMNS}}^{T}=L_{e}^{\dagger} M_{\nu} L_{e}^{*}, \quad V_{0} \equiv L_{D}^{\dagger} L_{e}
$$

$M_{\nu}^{\prime}$ is the light neutrino mass matrix in the basis where the charged leptons are diagonal. It can have the same forms as discussed in Section 3 for $M_{\nu}$. With the substitutions $M_{\nu} \rightarrow M_{\nu}^{\prime}$ and $L_{D} \rightarrow V_{0}$, the equations used above are unaltered.

\footnotetext{
${ }^{9}$ We will not discuss the origin of these lopsided matrices, which, e.g., can be due to family symmetries [7].
} 
The crucial difference is that the assumed form of $L_{D}$ in Eq. (3) does not necessarily apply to $V_{0}$ in the lopsided case. Since $V_{0}$ contains off-diagonal entries of order one, we may arrange for terms of equal order to cancel each other in $V_{0} M_{\nu}^{\prime} V_{0}^{T}$. This is not fine tuning because we are, in effect, cancelling an experimental term with a theoretical one, rather than cancelling two theoretical parameters against each other. To put it another way, we are simply using a theoretical term to generate an experimental parameter of the same order. The result is that we may be able to have a form for $R_{D}^{\dagger} M_{R}^{-1} R_{D}^{*}$ which does not have such a strong hierarchy, and which in turn may not imply the restrictive cascade form for $M_{D}$. In such a scenario, some or all of the large mixing in $V_{\text {PMNS }}$ comes from charged lepton unitary rotations.

To examine the lopsided case further we must see what can be said about the matrix $V_{0}$. Again, we can look at the CKM matrix for possible constraints. It can tell us about the potential lopsidedness in the down quark and charged lepton mass matrices. The operator we are using to generate lopsidedness contributes to $M_{e}$ as the transpose of its contribution to $M_{d}$, as is familiar from SU(5) models. Hence, large rotations in $L_{e}$ would coincide with large rotations in $R_{d}$ and vice versa. We now see that the experimental values are consistent with either a cascade structure or a lopsided structure for the down quark mass matrix in the third generation.

One might hope that the relatively large 1-2 mixing, which is consistent with a geometric hierarchy in the down quark matrix (cf. Section 5), would constrain the 1-2 mixing in $R_{d}$. This, however, turns out not to be the case. We can construct a matrix with all the desired features and generically large righthanded mixing, e.g.,

$$
M_{d} \sim\left(\begin{array}{ccc}
\frac{m_{d}}{m_{b}} & \frac{\sqrt{m_{d} m_{s}}}{m_{b}} & \frac{m_{d}}{m_{b}} \\
\frac{\sqrt{m_{d} m_{s}}}{m_{b}} & \frac{m_{s}}{m_{b}} & \frac{m_{s}}{m_{b}} \\
1 & 1 & 1
\end{array}\right), \quad L_{d} \sim\left(\begin{array}{ccc}
1 & \sqrt{\frac{m_{d}}{m_{s}}} & \frac{\sqrt{m_{d} m_{s}}}{m_{b}} \\
\sqrt{\frac{m_{d}}{m_{s}}} & 1 & \frac{m_{s}}{m_{b}} \\
\frac{\sqrt{m_{d} m_{s}}}{m_{b}} & \frac{m_{s}}{m_{b}} & 1
\end{array}\right), \quad R_{d} \sim\left(\begin{array}{ccc}
1 & 1 & 1 \\
1 & 1 & 1 \\
1 & 1 & 1
\end{array}\right) .
$$

Thus, although the CKM matrix is highly suggestive of either a partially cascade or lopsided form for the down quark mass matrix, it is difficult to constrain the form of $R_{d}$ and its counterpart $L_{e}$ in the latter case.

On the other hand, we note that since $V_{\text {PMNS }}$ has a small value for the (13) entry, naturalness requires that at least one entry in the column $L_{e}^{i 1}$ be correspondingly small. This suggests that we can rule out the extreme lopsided case shown above.

Lopsidedness also modifies the eigenvalue fitting we did in Section 6. Let us consider the case where $\mathbf{1 6}_{i} \mathbf{1 6}_{j} \mathbf{1 6}_{H} \mathbf{1 6}_{H}^{\prime}$ is lopsided and assume that it contributes to only one off-diagonal entry in $M_{d}$ and $M_{e}$ in a significant way. Then the mass matrices in Eq. (30) are modified to

$$
M_{d}=\left(\begin{array}{cc}
\alpha^{\prime}+\gamma^{\prime} & \alpha+\beta \\
\alpha-\beta+\gamma & 1
\end{array}\right), \quad M_{e}=\left(\begin{array}{cc}
\alpha^{\prime}+\gamma^{\prime} & \alpha-3 \beta+\gamma \\
\alpha+3 \beta & 1
\end{array}\right) \text {, }
$$

\footnotetext{
${ }^{10}$ This is simply due to the fact that $\mathbf{1 6}_{H}$ breaks $\mathrm{SO}(10)$ to $\mathrm{SU}(5)$, so $\mathbf{1 6}_{i} \mathbf{1 6}_{j} \mathbf{1 6} \mathbf{6}_{H} \mathbf{1 6}{ }_{H}^{\prime}$ is basically an $\mathrm{SU}(5)$ Yukawa operator for down quarks and charged fermions, suppressed by $v / M$.
} 
with the corresponding eigenvalues

$$
\frac{m_{s}}{m_{b}}=\left|\frac{\alpha^{\prime}+\gamma^{\prime}+\beta^{2}-\alpha^{2}-\gamma(\alpha+\beta)}{1+\gamma^{2}}\right|, \quad \frac{m_{\mu}}{m_{\tau}}=\left|\frac{\alpha^{\prime}+\gamma^{\prime}+9 \beta^{2}-\alpha^{2}-\gamma(\alpha+3 \beta)}{1+\gamma^{2}}\right| .
$$

This yields

$$
\frac{m_{\mu}}{m_{\tau}}-\frac{m_{s}}{m_{b}}=\frac{2 \beta(4 \beta-\gamma)}{1+\gamma^{2}} \stackrel{\gamma \sim 1}{\longrightarrow} \beta \sim 4 \times 10^{-2},
$$

which is only a slight improvement over the symmetric case, a cascade structure in $M_{D}$ is still inconsistent with the charged fermion hierarchies. Thus, in the absence of additional contributions to the mass matrices, it seems we must rely on large, lopsided mixing between the second and third generations to alleviate the need for a cascade strucure in the 2-3 block of $M_{D}$.

\section{$9 \quad$ Small Entries and Mixing}

Aside from lopsided matrices, there is another scenario in which $V_{0}$ can play an important role. We saw in Section 1 that the entries of the first row and column of $R_{D}^{\dagger} M_{R}^{-1} R_{D}^{*}$ are smaller in the cases 1 and 2. If we allow cancellations between these entries of order $\lambda$ and the mixing parameter $\mu^{\prime} \sqrt{\frac{\eta}{\epsilon}}$, we might expect some qualatatively different results. In this more general case, we use $\mu^{\prime}, \nu^{\prime}$ and $\rho^{\prime}$ to parameterize $V_{0}$ rather than $L_{D}$. Including the effects of $L_{e}$, we no longer have the symmetry constraints $(\mu, \nu, \rho) \sim\left(\mu^{\prime}, \nu^{\prime}, \rho^{\prime}\right)$.

We consider case 1:

$$
R_{D}^{\dagger} M_{R}^{-1} R_{D}^{*} \sim\left(\begin{array}{ccc}
\frac{\lambda}{\eta^{2}} & \frac{1}{\eta \epsilon}\left(\lambda+\mu^{\prime} \sqrt{\frac{\eta}{\epsilon}}\right) & \frac{1}{\eta}\left(\lambda+\mu^{\prime} \sqrt{\frac{\eta}{\epsilon}}\right) \\
\frac{1}{\eta \epsilon}\left(\lambda+\mu^{\prime} \sqrt{\frac{\eta}{\epsilon}}\right) & \frac{1}{\epsilon^{2}} & \frac{1}{\epsilon} \\
\frac{1}{\eta}\left(\lambda+\mu^{\prime} \sqrt{\frac{\eta}{\epsilon}}\right) & \frac{1}{\epsilon} & 1
\end{array}\right) .
$$

Here, although the unitary rotations remain relatively close to unity, the rotation parameter $\mu^{\prime} \sqrt{\frac{\eta}{\epsilon}}$ may be large enough to cancel the experimental term $\lambda$. Such cancellation is only possible if $\mu^{\prime} \sim 1$.⿴囗十 Under geometrical constraints, the (11) entry will be $\frac{\lambda}{\eta^{2}}$, while the (12) entry could be much smaller than $\frac{\lambda}{\eta \epsilon}$. One can proceed to analyze the mixing parameters in $R_{D}$ as in Section 4 . Due to the relatively large (11) entry, one finds that the constraints

$$
\mu \lesssim \sqrt{\frac{\eta}{\epsilon}}\left(1+\frac{\mu^{\prime}}{\lambda} \sqrt{\frac{\eta}{\epsilon}}\right), \quad \nu \lesssim \sqrt{\nu}\left(1+\frac{\mu^{\prime}}{\lambda} \sqrt{\frac{\eta}{\epsilon}}\right), \quad \rho \lesssim \sqrt{\epsilon}
$$

are required to preserve the small (12) and (13) entries. Since this requires $\mu \ll \mu^{\prime} \sim 1$, the charged lepton rotations would have to be significantly larger than those from the Dirac neutrino matrix, at least for the 1-2 mixing. This would suggest an approximately geometric structure in

\footnotetext{
${ }^{11}$ Since $\mu^{\prime}$ includes contributions from $L_{e}$, its coefficient $\sqrt{\eta / \epsilon}$ should be $\sqrt{m_{e} / m_{\mu}}$ if the charged lepton ratio is larger. However, since $\sqrt{m_{e} / m_{\mu}} \sim 0.1 \sim \sqrt{\eta / \epsilon}$ under our assumptions, we keep our familiar notation.
} 
the charged lepton matrix and a Dirac neutrino matrix with very small first generation mixing. Thus, the Dirac neutrino matrix would have a more restricted form than the cascade hierarchy derived for the simpler case without cancellations. Unless some additional information prompts us to favor these textures for $M_{\nu}, M_{D}$ and $M_{e}$, there is no compelling reason to further pursue this route.

In the second case, where $\left(M_{\nu}\right)_{11} \sim 0$, we find that the constraints are the same as those listed in Eq. (25), i.e., the same as we found for this case without allowing for cancellations. These results hold because, regardless of how small $\lambda+\mu^{\prime} \sqrt{\frac{\eta}{\epsilon}}$ may be, we retain the same relative hierarchy between the first generation entries and the same hierarchy in the 2-3 block, cf. Eq. (15).

We conclude that these potential cancellations have little effect on our previous considerations.

\section{Outlook}

Barring cancellations or additional flavor symmetries, the observed pattern of neutrino mass splittings and mixing angles leads us to two related propositions for simple model building in the general context of a grand-unified theory with type-I seesaw mechanism. The first is a double hierarchy, with respect to the hierarchy of the Dirac matrix, $M_{D}$, in the effective heavy neutrino matrix $M_{R}$. The second, contingent upon the first, is a cascade structure in $M_{D}$, or a texture which is even closer to diagonal. These conclusions follow only from the structure of the type-I seesaw formula, together with the observation that the experimental neutrino data most naturally arise from an approximately democratic effective light neutrino matrix. If the neutrino masses obey a normal hierarchy, i.e., $m_{1} \lesssim m_{2} \sim \sqrt{\Delta m_{\text {sol }}^{2}} \ll m_{3} \sim \sqrt{\Delta m_{\text {atm }}^{2}}$, it is possible to relax these constraints, but it remains true that $M_{R}$ should have an enhanced hierarchy and $M_{D}$ should have a sub-geometrical structure. Moreover, in this case some approximate symmetry must exist to generate a second large mixing angle and a hierarchy consistent with experiment.

These conclusions are rather general and not restricted to the specific model with small representations outlined in Section 2. They hold for hierarchical, symmetric matrices, up to factors of order one. In light of the quark and charged lepton mass hierarchies, it is natural for $M_{D}$ to be hierarchical. In particular, this matrix is closely related to the up quark matrix in many GUT models. Family symmetries will also tend to engender such relations. In Section 7 we showed that even adding an operator which ostensibly only contributes to the Dirac neutrino matrix does not necessarily relax our conclusions.

Can we implement these textures in a complete model? We discussed a scenario with a $U(1) \times \mathbb{Z}_{2} \times \mathbb{Z}_{2}$ flavor symmetry, where we generated a cascade structure for the Dirac matrices through the Froggatt-Nielsen mechanism. A double hierarchy in $M_{R}$ is natural if it is an effective operator generated by integrating out singlets coupled to $\mathbf{1 6}_{i} \overline{\mathbf{1 6}}_{H}$, where this coupling has an eigenvalue hierarchy similar to that in $M_{D}$. However, this structure lead to problems in the quark sector. We have seen that the relatively large Cabibbo angle implies that the down quark matrix 
is not purely cascade-like, although a cascade structure in the third generation is supported. This does not necessarily conflict with a fully cascade pattern in $M_{D}$, but it requires a somewhat more complicated picture than the simple model described above. Furthermore, in our specific model, we rely on the antisymmetric operator $\mathbf{1 6}_{i} \mathbf{1 6}_{j} \mathbf{1 0}_{H} \mathbf{4 5} \mathbf{5}_{H}$ to differentiate the down quark and charged lepton matrices. This implies that its contributions cannot be too small. Since it also contributes to the up-quark and neutrino matrices, it becomes difficult to reconcile a cascade structure in these matrices with the strong up-quark hierarchy in a natural way.

Lopsided models may provide us a way out of these potential difficulties. Compared with a cascade pattern, they are equally compatible with the CKM matrix. For the purposes of mass fitting, lopsidedness slightly relaxes the need for large off-diagonal contributions from $\mathbf{1 6}_{i} \mathbf{1 6}_{j} \mathbf{1 0}_{H} \mathbf{4 5}_{H}$. More importantly, a lopsided charged lepton matrix introduces large rotations which contribute to the PMNS matrix. If these are primarily responsible for one or both of the large mixing angles, it is possible to reduce the pull towards a double hierarchy in $M_{R}$. This in turn can relax the constraints that lead us to a cascade structure for $M_{D}$ and so for $M_{u}$. Exactly how much lopsidedness can obviate the need for a double hierarchy remains an open question. The atmospheric mass splitting remains small compared to the quark mass splittings, irrespective of the origin of the large mixing angles. This will tend to require an enhanced hierarchy in at least part of $M_{R}$. Additionally, while it is technically possible that most or all of the PMNS structure comes from charged lepton rotations, we must ask how much can be done in a natural way. For example, as discussed at the end of Section 8, a small value for $\theta_{13}$ precludes generically large mixing from lopsidedness in all generations.

This brings us to the nature and origin of $\theta_{13}$ in general, which we have not addressed in detail in this paper. We chose to leave this an open question in light of the current uncertainty in the size of $\theta_{13}$ : only an upper bound is known. While it is clear that the solar and atmospheric mixing angles are large compared with those in the quark sector, $\theta_{13}$ may or may not be comparatively small. Actually, the experimental upper limit, approximately $10^{\circ}$, is of the same order as the Cabibbo angle. This is large enough that its smallness compared to the other neutrino angles may be explained by normal fluctuations of order one parameters without violating our sense of naturalness [6]. However, if $\theta_{13}$ is significantly closer to zero we should seek some more robust explanation. For the forms of $M_{\nu}$ listed in Section 3, this would require a symmetry closely relating various matrix elements. Another possibility arises for partially lopsided matrices: if one large mixing angle arises from the charged lepton sector and the other from $M_{\nu}$ then it is natural to preserve a small third angle. Clearly, it is important to determine the order of $\theta_{13}$.

In summary, a combination of partially lopsided and partially cascade matrices, in conjunction with an enhanced hierarchy in $M_{R}$, seems to be the most natural route to explain the generic features of the quark and lepton data in a grand-unified model. The details of a complete model remain to be worked out, but our conclusions follow from a fairly general framework. It will be interesting to see if a workable model can be obtained with relatively simple family symmetries and what consequences there might be for experimental predictions. 
We would like to thank S. Willenbrock for useful comments on the manuscript. This work was supported in part by the U. S. Department of Energy under contract No. DE-FG02-91ER40677, as well as the Sonderforschungsbereich Transregio 9 Computergestützte Theoretische Teilchenphysik of the Deutsche Forschungsmeinschaft.

\section{A Derivation of Expanded Seesaw Formula}

In this appendix, we derive the extended seesaw formula, given in Eq. (38). As mentioned in Section 0 , it is crucial that terms quadratic in $\bar{M}^{\prime} \bar{M}^{-1}$ do not appear. The formula was originally derived in Ref. [19] through a slightly different calculation.

As displayed in Eq. (35), we propose the operators

$$
W_{S}=\bar{M}_{i j} \mathbf{1 6} \overline{\mathbf{1 6}}_{H} \mathbf{S}_{j}+\bar{M}_{i j}^{\prime} \mathbf{1 6} \overline{\mathbf{1 6}}_{H}^{\prime} \mathbf{S}_{j}+m_{s}\left(M_{S}\right)_{i j} \mathbf{S}_{i} \mathbf{S}_{j}
$$

We integrate out the singlet fields, $\mathbf{S}$, by taking a partial derivative and setting it equal to zero,

$$
\frac{\partial W_{S}}{\partial S_{j}} \equiv 0: \quad \mathbf{S}_{i}=-\frac{1}{2 m_{s}}\left[\bar{M}_{i j} \mathbf{1 6} \overline{\mathbf{1 6}}_{H}+\bar{M}_{i j}^{\prime} \mathbf{1 6}_{i} \overline{\mathbf{1 6}}_{H}^{\prime}\right]\left(M_{S}^{-1}\right)_{j k} .
$$

Plugging this into our initial equation yields

$$
\begin{aligned}
W_{S}^{\mathrm{eff}}=-\frac{1}{4 m_{s}} \mathbf{1 6} \mathbf{6}_{i}\left[\left(\bar{M} M_{S}^{-1} \bar{M}^{T}\right)_{i j} \overline{\mathbf{1 6}}_{H} \overline{\mathbf{1 6}}_{H}\right. & +\left(\bar{M}^{\prime} M_{S}^{-1} \bar{M}^{\prime T}\right)_{i j} \overline{\mathbf{1 6}}_{H}^{\prime} \overline{\mathbf{1 6}}_{H}^{\prime} \\
& \left.+2\left(\bar{M}^{\prime} M_{S}^{-1} \bar{M}^{T}\right)_{i j} \overline{\mathbf{1 6}}_{H} \overline{\mathbf{1 6}}_{H}^{\prime}\right] \mathbf{1 6}{ }_{j}
\end{aligned}
$$

Now we let the Higgs fields acquire their GUT and weak scale vevs and we include the Dirac term $\nu M_{D} N$, where $\nu$ and $N$ are the left and right-handed neutrinos, respectively. Suppressing the generation indices, we obtain

$$
\begin{aligned}
W_{N}= & -\frac{1}{4 m_{s}}\left[v^{2} N\left(\bar{M} M_{S}^{-1} \bar{M}^{T}\right) N+u^{\prime 2} \nu\left(\bar{M}^{\prime} M_{S}^{-1} \bar{M}^{\prime T}\right) \nu+2 u^{\prime} v \nu\left(\bar{M}^{\prime} M_{S}^{-1} \bar{M}^{T}\right) N\right] \\
& +u \nu M_{D} N
\end{aligned}
$$

We extremize with respect to $N$ and find

$$
\frac{\partial W_{N}}{\partial N} \equiv 0: \quad N=\left[\frac{u m_{s}}{v^{2}} M_{D}\left(\bar{M}^{-1}\right)^{T} M_{S} \bar{M}^{-1}-\frac{u^{\prime}}{v} \bar{M}^{\prime} \bar{M}^{-1}\right] \nu .
$$

Inserting this into the last equation and performing a little algebra gives the amended seesaw formula

$$
W_{\nu}^{\mathrm{eff}} \simeq\left\{M_{D}\left(\bar{M}^{-1}\right)^{T} M_{S} \bar{M}^{-1} M_{D}^{T}-\frac{1}{2} \frac{u^{\prime} v}{u m_{s}}\left[\bar{M}^{\prime} \bar{M}^{-1} M_{D}^{T}+M_{D}\left(\bar{M}^{\prime} \bar{M}^{-1}\right)^{T}\right]\right\} \frac{u^{2} m_{s}}{v^{2}} .
$$




\section{References}

[1] W. M. Yao et al. [Particle Data Group], J. Phys. G 33, 1 (2006).

[2] A. Strumia and F. Vissani, arXiv:hep-ph/0606054.

[3] P. Minkowski, Phys. Lett. B 67, 421 (1977).

[4] H. Georgi, in: Particles and fields (ed. C. Carlson), AIP Conf. Proc. 23, 575 (1975);

H. Fritzsch and P. Minkowski, Annals Phys. 93 (1975) 193.

[5] R. N. Mohapatra and A. Y. Smirnov, Ann. Rev. Nucl. Part. Sci. 56, 569 (2006);

G. Altarelli, arXiv:0705.0860 [hep-ph].

[6] G. Altarelli and F. Feruglio, New J. Phys. 6, 106 (2004).

[7] C. H. Albright and S. M. Barr, Phys. Rev. D 62, 093008 (2000); Phys. Rev. D 64, 073010 (2001).

[8] see e.g., K. S. Babu, J. C. Pati and F. Wilczek, Nucl. Phys. B 566, 33 (2000);

R. Dermisek and S. Raby, Phys. Rev. D 62, 015007 (2000);

T. Blazek, S. Raby and K. Tobe, Phys. Rev. D 62, 055001 (2000);

J. Sayre and S. Wiesenfeldt, Phys. Lett. B 637, 295 (2006).

[9] K. S. Babu and R. N. Mohapatra, Phys. Rev. Lett. 74, 2418 (1995).

[10] I. Dorsner and S. M. Barr, Nucl. Phys. B 617, 493 (2001).

[11] C. D. Froggatt and H. B. Nielsen, Nucl. Phys. B 147, 277 (1979).

[12] G. Anderson, S. Raby, S. Dimopoulos, L. J. Hall and G. D. Starkman, Phys. Rev. D 49, 3660 (1994);

S. Wiesenfeldt and S. Willenbrock, arXiv:0707.3300 [hep-ph].

[13] For a recent brief review, see A. Melfo, AIP Conf. Proc. 917, 252 (2007).

[14] J. Lesgourgues and S. Pastor, Phys. Rept. 429, 307 (2006);

S. Hannestad and G. G. Raffelt, JCAP 0611, 016 (2006).

[15] P. F. Harrison, D. H. Perkins and W. G. Scott, Phys. Lett. B 530, 167 (2002).

[16] S. Antusch, J. Kersten, M. Lindner, M. Ratz and M. A. Schmidt, JHEP 0503, 024 (2005).

[17] H. Harari, H. Haut and J. Weyers, Phys. Lett. B 78, 459 (1978);

L. Lavoura, Phys. Lett. B 228, 245 (1989);

H. Fritzsch and J. Plankl, Phys. Lett. B 237, 451 (1990).

[18] G. Altarelli, F. Feruglio and I. Masina, Phys. Lett. B 472, 382 (2000).

[19] S. M. Barr, Phys. Rev. Lett. 92, 101601 (2004).

[20] H. Fritzsch, Phys. Lett. B 70, 436 (1977).

[21] Y. Nomura and T. Yanagida, Phys. Rev. D 59, 017303 (1999);

T. Asaka, Phys. Lett. B 562, 291 (2003);

W. Buchmuller, L. Covi, D. Emmanuel-Costa and S. Wiesenfeldt, arXiv:0709.4650 [hep-ph]. 